\title{
Clinical and In-Silico study of COVID-19 patients using Thymus capitatus extract as adjuvant Therapy
}

\author{
Abdul M Gbaj ${ }^{*}$, Inass A Sadawe ${ }^{1}$, Nisreen H Meiqal ${ }^{1}$, Salah M Bensaber ${ }^{1}$, Abdulhakim M Qabaj ${ }^{2}$, Abdulhamid \\ R Hamid $^{3}$ Massaud Salem Maamar ${ }^{4}$ and Anton Hermann ${ }^{5}$
}

${ }^{1}$ Department of Medicinal Chemistry, Faculty of Pharmacy, University of Tripoli, Libya

${ }^{2}$ Arada polylinic, Arada, Tripoli Libya

3 Judicial Expertise and Research Centre, Tripoli, Libya

${ }^{4}$ Zoology Department, Faculty of Science, Tripoli University, Libya

${ }^{5}$ Department of Biosciences, University of Salzburg, Salzburg, Austria

*Corresponding author: Abdul M Gbaj, Professor of Genetics and Biochemistry, Department of Medicinal Chemistry, Faculty of Pharmacy, University of Tripoli, Libya.

Received Date: Decembe 11, 2020

Published Date: February 08, 2021

\begin{abstract}
Thymus capitatus extract has been considered a promise as antiviral agent against COVID-19 viruses. We hypothesized that Thymus capitatuscomponents may interact with key protein targets of COVID-19 (coronavirus 2 (SARS-CoV-2) causing severe acute respiratory syndromes. Molecular docking analysis was carried out using 31 components of Thymus capitatus with SARS-CoV-2 protease enzyme (6LU7) and spike glycoprotein (6VSB). The compounds with the best normalized docking scores to protease enzyme were allo-Aromadendrene (-6.3 kcal/mole), spathulenol $(-6.6 \mathrm{kcal} / \mathrm{mole})$ and ledene $(-6.8 \mathrm{kcal} / \mathrm{mole})$. The best docking ligands for spike glycoprotein were allo-Aromadendrene (-6.6 kcal/ mole), spathulenol (-6.6 kcal/mole) and ledene ( $-7.3 \mathrm{kcal} / \mathrm{mole})$. All Thymus capitatus components may act synergistically to produce the therapeutic action. Thymus capitatus components may potentiate the effect of prednisolone, azithromycin and other medicines used to treat COVID-19 patients.
\end{abstract}

Key words: COVID-19; corona virus; molecular docking; antiviral; Thymus capitatus

\section{Introduction}

The outbreak of Coronavirus SARS-Cov-2 disease (COVID-19) in Wuhan (China) in December 2019 and its worldwide spread has led in some cases to heavy disease symptoms finally causing deaths [1,2]. During the year 2020, the disease has affected almost all countries on the globe. The majority of people infected with the COVID-19 virus face mild medium respiratory symptoms and recover mostly without any treatment [3,4]. Elderly people and people with chronic medical conditions such as diabetes, chronic respiratory disease, cardiovascular disease, or cancer are expected to develop more serious symptoms [5,6]. Young people and adults who have no serious medical conditions are requested to self-manage their symptoms in isolation possibly taking a pain killer such as paracetamol or using hyperthermia and lifestyle modifications such as increased rest and taking good supply of water $[7,8]$. However, most of the present procedures do not provide adequate medical advice how to treat cough and other symptoms $[9,10]$. COVID-19 symptoms usually start with dry cough, fever, and dyspnea, which is similar to other viral respiratory diseases such as ordinary flu. Consequently, the diagnosis of COVID-19 becomes difficult. Generally, the incubation period of COVID-19 is approximately a few to 15 days. SARS-Cov- 2 has a dramatic transmission capability $[9,10]$. This could be related to the fact that 
a significant number of patients are asymptomatic (up to 60\%) who accidentally participate to the spread of the disease $[11,12]$. In COVID-19 the infection from mild upper respiratory tract problems to severe acute respiratory syndromes which may lead to respiratory failure and shock which in addition may cause multiple organ failure. Other accompanying features might arise such as fatigue, diarrhea, headache, or lymphopenia and cardiovascular symptoms $[13,14]$.

Corticosteroids, as generally used immunosuppressants and anti-inflammatory agents play an significant role in the treatment of critical diseases $[15,16]$. Previous studies showed that corticosteroid was used to treat coronavirus diseases, including Middle East respiratory syndrome (MERS) and severe acute respiratory syndrome (SARS) [17]. Studies have demonstrated that cytokine reactions caused by the enormous release of cytokines were major cause of death in a number of cases of SARS and MER $[18,19]$ and corticosteroids could have a positive antagonistic effect on the cytokine storm [20]. Azithromycin has immunomodulatory and antiviral properties that might be of attention in the treatment of COVID-19. The use of this macrolide has been connected with the progression of clinical therapy in other viral infections and community acquired pneumonia [21].

Azithromycin has been shown to exert in vitro activity against SARS-CoV-2 and may operate at many points of the viral cycle. Additionally, the immunomodulation of Azithromycin improved clinical outcomes by its capability to preserve epithelial integrity, down regulate cytokine production and avoid lung fibrosis and hence could play a role in the hyperinflammatory stage during COVID-19 infection [22,23].

Current confirmation showed that nutritional supplementation might have a supportive role in COVID-19 patients. Administration of higher than recommended daily doses of nutrients such as vitamins $\mathrm{C}, \mathrm{D}, \mathrm{E}$, omega-3 fatty acids and Zinc exert a valuable effect by significantly reducing SARS-CoV-2 viral load and the time of hospitalization [24,25]. These nutrients are known for their immunomodulatory and antioxidant ability. Deficiencies of such nutrients could lead to immune dysfunction and enhance vulnerability to pathological infection. Dietary deficiency of vitamins and minerals has been found in high-risk groups of COVID-19 patients, such as the elderly by increasing morbidity and risk of mortality [26]. Natural products and herbal medicine are employed to treat respiratory infection, and several have been approved as drugs, over-the-counter or food additives. The usual low to negligible toxicity makes herbal medicines and natural product reasonable prophylactic candidates for treatment. Using in silico technology to study natural products has been found utterly effective to find blockers of enzyme function and membrane receptors of human coronavirus [27,28]. The symptoms of COVID-19 are characteristic exhibiting uncontrolled inflammation, such cytokine release syndrome, and hence anti-inflammatory herbs are able to suppress such often deadly symptoms. Digestive enzymes, low $\mathrm{pH}$ in the gastric vicinity or the gut microbiome have less effect on the bioavailability of natural products and herbs in comparison to antibody and other prophylactics [29,30].

Thyme is a plant with a long history in traditional medicine in Libya that has antiseptic, antimicrobial, and antiviral properties. It also has useful effects on the cardiovascular and respiratory system [31-33]. Thymus capitatus belongs to the thymus genus and the Lamiaceae family. This plant is small, perennial and grows in hot and dry climates like Libya, Tunisia, Algeria etc [34,35]. Thymus capitatus contains bioactive compounds such as phenols, phenolic acids, terpenes and flavonoids [36-38]. Thymus capitatus is containing a rich source of vitamins, particularly vitamin $C$, vitamin A, and vitamin B6. Furthermore, in the leaves of this plant, minerals such as calcium, iron, potassium, manganese, magnesium, and selenium are found in abundance [39].

Molecular modeling is a recognized computational tool to aid early drug discovery and development. It is used to generate ideas of a compounds or macromolecules 3D conformation, proteinligand interactions, and allows forecasts about biological activities. The integration of molecular modeling in drug or vaccine design can help in early drug or vaccine discoveries [40-42].

The main aim of this study was to investigate the effects of Thymus Capitatus extract on COVID-19 patients clinically to provide information if it can be used as a clinically compatible option for adjuvant therapy. We also performed in-silico studies on two targets -protease enzyme and spike glycoprotein - to collect information if they are suited as targets by computational drug repurposing procedures to allocate appropriate inhibitory agents.

\section{Materials and Methods}

\section{Clinical evaluation}

The study was performed between October 1st and November 30th, 2020, a total of 60 cases were investigated at Arada polyclinic, Tripoli Libya. All the patients were informed and consented. Sixty COVID-19 patients were enrolled in the study. The age group of patients was from 40-85 years. The patients were treated at the Out Patient Department (OPD) of Arada polyclinic, Arada, Tripoli Libya.

All patients were screened for COVID-19 symptoms by polymerase-chain reaction (PCR) as well IgM and IgG antibody tests within 5 days of the study. Additionally, a history of diabetes, chronic obstructive pulmonary disease COPD, coronary artery disease CAD, and use of immunosuppressants were recorded for risk evaluation. All patients came to the clinic with pulmonary infiltrate bilateral atypical pneumonia (walking pneumonia). Patients diagnosed with clinical symptoms showed: sore throat (pharyngitis), felt tired (fatigue), had chest pain, mild to severe chills, high-grade fever, persistent cough that can be dry or produce colored mucus, sneezing dyspnea and severe headache. In addition, high-resolution 
computed tomography HRCT, also called thin-section scan (CT scanning) was used to confirm pneumonia diagnosis.

The patients were divided into two groups: the first group consisted of 60 patients (45 males and 15 females) to whom Thymus capitatus $(10 \mathrm{~g} / 200 \mathrm{ml}$ boiled water containing the $10 \mathrm{~g}$ of stem and leaves of Thymus capitatus) was administered. In addition, patients were given prednisolon $40 \mathrm{mg}$ daily for 7 days and then the dose gradually decreased by $10 \mathrm{mg}$ every 7 days until the last dosage of $10 \mathrm{mg}$ for 7 days. Azitromycine $500 \mathrm{mg}$ tablets were used once a day for 6 days. Ceftriaxon (Rocephin $1 \mathrm{~g}$ powder for injection solution) was given intramuscular twice a day for a week. Ipratropium $(250 \mu \mathrm{g} / 1 \mathrm{ml})$ and salbutamol $(5 \mathrm{mg} / 2.5 \mathrm{ml})$ were inhaled using a nebulizer every 8 hours for two weeks. The patients were also treated with standard prophylactic LMWH Nadroparin calcium (Fraxiparine injection ${ }^{\circledR}$ ) subcutaneously as a single daily dose of $0.3 \mathrm{ml}(2,850 \mathrm{IU})$ or $0.6 \mathrm{ml}(5700 \mathrm{IU} / 0.6 \mathrm{~mL})$ for at least 7 days dosage. In addition, one tablet daily of Vitamin $\mathrm{C}$ (Ascorbic Acid) $500 \mathrm{mg}$, Vitamin D (from Cholecalciferol) $10 \mu \mathrm{g}$ and zinc $15 \mathrm{mg}$ were supplied for two months.

\section{Molecular docking}

The starting geometry of Thymus capitatus constituents was downloaded from Pubchem (https://pubchem.ncbi.nlm.nih.gov) into Chem3D Ultra software (version 8.0, Cambridge soft Com., USA). The optimized geometry of Thymus capitatus constituents with the lowest energy was used for molecular dockings. The crystal structure of COVID-19 main protease in complex with an inhibitor N3 (6LU7) was downloaded from the Protein Data Bank http://www.rcsb.org/structure/6LU7 and prefusion 2019-nCoV spike glycoprotein with a single receptor-binding domain (6VSB) was downloaded from the Protein Data Bank http://www.rcsb. org/structure/6VSB. Molecular dockings of Thymus capitatus constituents with 6LU7 and 6VSB were accomplished by Auto Dock 4.2 software from Scripps Research Institute (TSRI) (http:// autodock.scripps.edu/). Firstly, polar hydrogen atoms were added into protein molecules. Then, partial atomic charges were calculated using Kollman methods for protease enzymes and spike glycoprotein with Thymus capitatus constituents [43]. In the process of molecular docking, the grid maps of dimensions: (60尺 X $60 \AA \mathrm{X}$

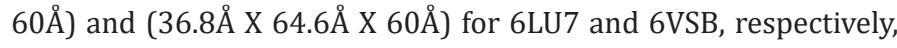
with a grid-point spacing of $0.376 \AA$ and the grid boxes centered were used [44]. The number of genetic algorithm runs and the number of evaluations were set to 100 . All other parameters were default settings. Cluster analysis was performed on basis of docking results by using a root mean square (RMS) tolerance of $2.0 \AA$, dependent on the binding free energy. Lastly, the dominating configuration of the binding complex of Thymus capitatus constituents with protease enzyme and spike glycoprotein fragments with minimum binging energy were determined which relied strongly on the information of 3D-structures of the protease enzyme and spike glycoprotein binding site and ultimately generated a series of protease enzyme- binding complexes and spike glycoprotein-binding complexes.

\section{Results and Discussion}

\section{Clinical spectrum}

Patients who had recovered from COVID-19, 95.0\% reported persistence of at least 1 symptom, particularly very mild dyspnea and mild fatigue. 5\% (3 patients) died a 84 years old man with diabetes and hypertension, a lady 72 years old with myopathy and a 45 years man. Limitations of the study include the lack of information on symptom history before acute COVID-19 illness and the lack of details on symptom severity. Furthermore, this is a single-center study with a relatively small number of patients (60) and without a control group of patients who did not use Thymus capitatus.

Thymus capitatus contains a variety of functional chemical groups including phenols, phenolic acids, terpenes and flavonoids. The most important compounds in Thymus capitatus include 4-thujanol, allo-Aromadendrene, $\alpha$-copaene, $\alpha$-pinene, $\alpha$-terpineol, $\beta$-bisabolene, bronyl, $\beta$-phellandrene, $\beta$-pinene, carvacrol, carvenone, eugenol, cis-carveol, $\beta$-cis-ocimene, limonene, geraniol, neral, spathulenol, linalool, p-cymene, thymol, terpiene4-ol, terpinolene, p-cymen-8-ol, trans-piperitol, carvotanacetone, cis-dihydrocarvone, champhene, $\alpha$-humulene, 3-octanol and ledene (viridiflorene). For example, thymol exhibits numerous biological effects such as antioxidant, anti-inflammatory, free radical scavenging properties and immunomodulating properties. Also, carvacrol exhibits antimicrobial, antioxidant activities and anticarcinogenic effects [45]. Furthermore, many studies have confirmed the effect of thymol and carvacrol decreasing the proinflammatory cytokines: Interleukin (IL)-1 $\beta$, IL-6, and tumor necrosis factor (TNF)- $\alpha[46,47]$.

It is reported that in SARS-CoV-2 infection, the level of AngII increases [48]. The binding of AngII to AngII type 1 receptors (AT1R) is a vital mediator for majority of pro-inflammatory effects. The AngII type 2 receptor (AT2R) is activated by AngII which activates some inflammatory cells. AT1R activation induces the overexpression of chemokines, adhesion molecules, and cytokines via special pathways such as NF-kB up-regulation. NF-kB plays an essential role in the regulation of numerous pro-inflammatory genes $[49,50]$. It has been reported that herbal thyme acts as a negative regulator of NF-kB. Furthermore, other studies showed that the plasma levels of most pro-inflammatory cytokines, such as TNF, IL1B, IL7, IL1RA, IL8, IL9, IL10, basic fibroblast growth factor (FGF), and other pro-inflammatory factors were increased in COVID-19. Consequently, thyme fits as an inhibitory agent of proinflammatory cytokines [51,52]. Hence, Thymus capitatus can be considered as adjuvant therapy for COVID-19 as described in the literature [53,54].

Thymus capitatus contains many phenolic compounds such as thymol and carvacrol which were used traditionally in Libya 
to treat chronic bronchitis, sore throat, cough, asthma, colds, and other respiratory infections. Thyme is an expectorant, antiseptic, and antispasmodic herbal medicine with respiratory clearance nature [55]. During COVID-19 infection, acute lung injury and acute respiratory distress syndrome are the major reason for COVID-19 respiratory mortality. Damage of epithelial and endothelial respiratory cells, neutrophil infiltration, an extendedrelease of pro-inflammatory mediators, and pulmonary edema are characteristic for COVID-19 patients. Severe COVID-19 patients are characterized by a cytokine storm with increased serum levels of pro-inflammatory cytokines [56]. Cytokines such as TNF- $\alpha$ and IL-6 activate neutrophils and other inflammatory cells in the lung. Cytokine release leads to respiratory endothelial dysfunction which results in microvascular permeability and protein escape proceeded by hypoxemia. TNF- $\alpha$ and IL- 6 are components of an inflammatory response and have been shown to encourage the incidence of acute lung injury and acute respiratory distress syndrome [57]. Some studies $[46 ; 47]$ indicate the immunomodulatory effect of thymol in lipopolysaccharide-induced acute respiratory distress syndrome through TNF- $\alpha$ and IL-6 suppression in the broncho-alveolar lavage fluid (BALF). NF- $\mathrm{KB}$, a protein complex that triggers a proinflammatory signaling pathway in the lung leading to cytokine release is overexpressed in acute lung injury and acute respiratory distress syndrome $[57,58]$. Studies have shown that thyme extract is a negative regulator of NF-kB p65 and NF-kB p52 transcription factors whereby pro-inflammatory cytokines (IL-1 beta and IL-8) are reduced. Further studies also established that thyme extract acts as an inhibitor of cytokines IL-1-beta and IL-8, at both the mRNA and the protein level in normal human epithelial cells [59]. Thymol can also decrease the recruitment of inflammatory cells like neutrophils into bronchoalveolar lavage fluid. Thymol inhibits NF- $\mathrm{BB}$ activation [59] which is a transcription regulator of inflammatory and immune response genes [59] and hence can decrease the severity of acute lung injury and acute respiratory distress syndrome. Carvacrol, another active constituent of thyme has been also shown to have a therapeutic effect on acute lung injury and acute respiratory distress syndrome. The remedial effect of carvacrol on the acute respiratory distress syndrome is through decreasing inflammatory cytokines, reducing the number of inflammatory cells in bronchoalveolar lavage fluid, and by inhibition of NF- $\kappa \mathrm{B}[60,61]$. As a result, Thymus capitatus extract is suggested to have an ameliorating effect on respiratory symptoms of COVID-19 patients. The most common outline of coagulopathy seen in patients with COVID-19 is characterized by elevation in fibrinogen and D-dimer levels, and mild prolongation of prothrombin time/ activated partial thromboplastin time. Thymol has antiaggregant ability and it is able to inhibit platelet aggregation induced by thrombin, collagen, adenosine diphosphate and arachidonic acid [62].

In Libya some people use Thymus capitatus extract as a remedy for hypertension and other cardiovascular diseases.
Thymus capitatus has effective components such as thymol and carvacrol against oxidative agents causing cardiovascular disease [63]. Cardiovascular damages contribute to the morbidity of COVID-19 patients. Patients with cardiovascular disease (CVDs) are in addition susceptible to develop severe COVID-19 symptoms, in particular acute myocardial injury $[64,65]$. SARS-COV-22 appears able to provoke myocardial injury via a cytokine storm connected with a rigorous response by type 1 and type $2 \mathrm{~T}$ helper cells [64,65]. Furthermore, COVID-19-induced hypoxemia [66] and respiratory collapse could lead to myocardial dysfunction $[64,65]$. Cytokine storms could provoke multiple organ failure and increase inflammatory chemokines such as IL-6, IL-2, IL7 , interferon- $\gamma$, (TNF)- $\alpha$. Cardiovascular injury correlates with elevated inflammatory markers [64,65]. Moreover, the oxidative stress generated by SARS-CoV-2 might be a source of heart injures $[64,65]$. Thymus capitatus contains antioxidant components, and studies have shown that an appropriate dose of antioxidants can improve heart damage caused by COVID-19 infection [67]. It has been also demonstrated that thymol and carvacrol, derivatives of Thymus capitatus, have anti-inflammatory effects [68;69]. Taken together it appears that Thymus capitatus and its derivatives can protect cardiovascular damages induced by SARS-COV-22.

\section{Molecular docking analysis}

Table 1 shows the binding energies of Thymus capitatus constituents with SARS-CoV-2 protease enzyme (6LU7) and spike glycoprotein (6VSB) obtained by the molecular docking strategy. Molecular docking of Thymus capitatus constituents with SARSCoV-2 protease enzyme (6LU7) and spike glycoprotein (6VSB) were performed using Auto Dock 4.2. Thymus capitatus constituents and protease enzyme (6LU7) and spike glycoprotein (6VSB). Thymus capitatus constituents and protease enzyme (6LU7) and spike glycoprotein (6VSB) were kept as flexible molecules and were docked onto seven forms of protease enzyme (6LU7) and spike glycoprotein (6VSB) to obtain the privileged binding site of Thymus capitatus constituents. The molecular docking results are shown in Table 1. The modeling studies indicate van der Waals, hydrogen bonding (Table 1) and electrostatic interactions between Thymus capitatus constituents with protease enzyme (6LU7) and spike glycoprotein (6VSB). The contribution of van der Waals and hydrogen bonding interaction is much greater than that of electrostatic interaction because the sum of van der Waals energy, hydrogen bonding energy and desolvation free energy is larger than the electrostatic energy [70,71]. The Thymus capitatus constituents ledene and protease enzyme (6LU7) interactions are shown in Figure 1. The hydrophobic interaction of ledene with phe294 is the observed tendency of the nonpolar ability of ledene to aggregate in an aqueous solution and exclude water molecules around the protease enzyme. Ledene causes a segregation of water and nonpolar substances, which maximizes hydrogen bonding between water molecules and minimizes the area of contact between water and ledene molecules. In terms of 
thermodynamics, the hydrophobic effect is the free energy change of water surrounding a ledene molecule. The positive free energy change of the surrounding solvent designates hydrophobicity, while a negative free energy change implies hydrophilicity. The highest binding energies for Thymus capitatus constituents with protease enzyme (6LU7) were $-6.8,-6.6$ and $-6.3 \mathrm{kcal} / \mathrm{mol}$ for viridifloren, spathulenol and allo-aromadendrene, respectively. The highest binding energies of Sesquiterpenes for Thymus capitatus constituents with spike glycoprotein (6VSB) were -7.3, -6.6 and -6.6 $\mathrm{kcal} / \mathrm{mol}$ for viridifloren, spathulenol and allo-aromadendrene, respectively. Sesquiterpenes are lipophilic in nature and have a high $\log \mathrm{P}$ value indicating the significance of lipophilicity for the interaction with the active site. Viridiflorene, spathulenol and allo- aromadendrene are carbotricyclic sesquiterpenes and studies have shown that many carbocation intermediates in terpenoid cyclization cascades are subject to electron delocalization through bond coupling or through hyperconjugation and this directs the cyclization trajectory. Carbocation intermediates of viridiflorene are stabilized in the active site of a terpenoid cyclase by weakly polar interactions involving charge-dipole, charge-charge, and charge-quadrupole interactions with properly oriented amino acid side chains. In addition, carbocation intermediates can be stabilized by considerable partial negative charges generated by a ring $\pi$ electrons of the aromatic side chains of tyrosine, phenylalanine, and tryptophan through cation- $\pi$ interactions [72-74].

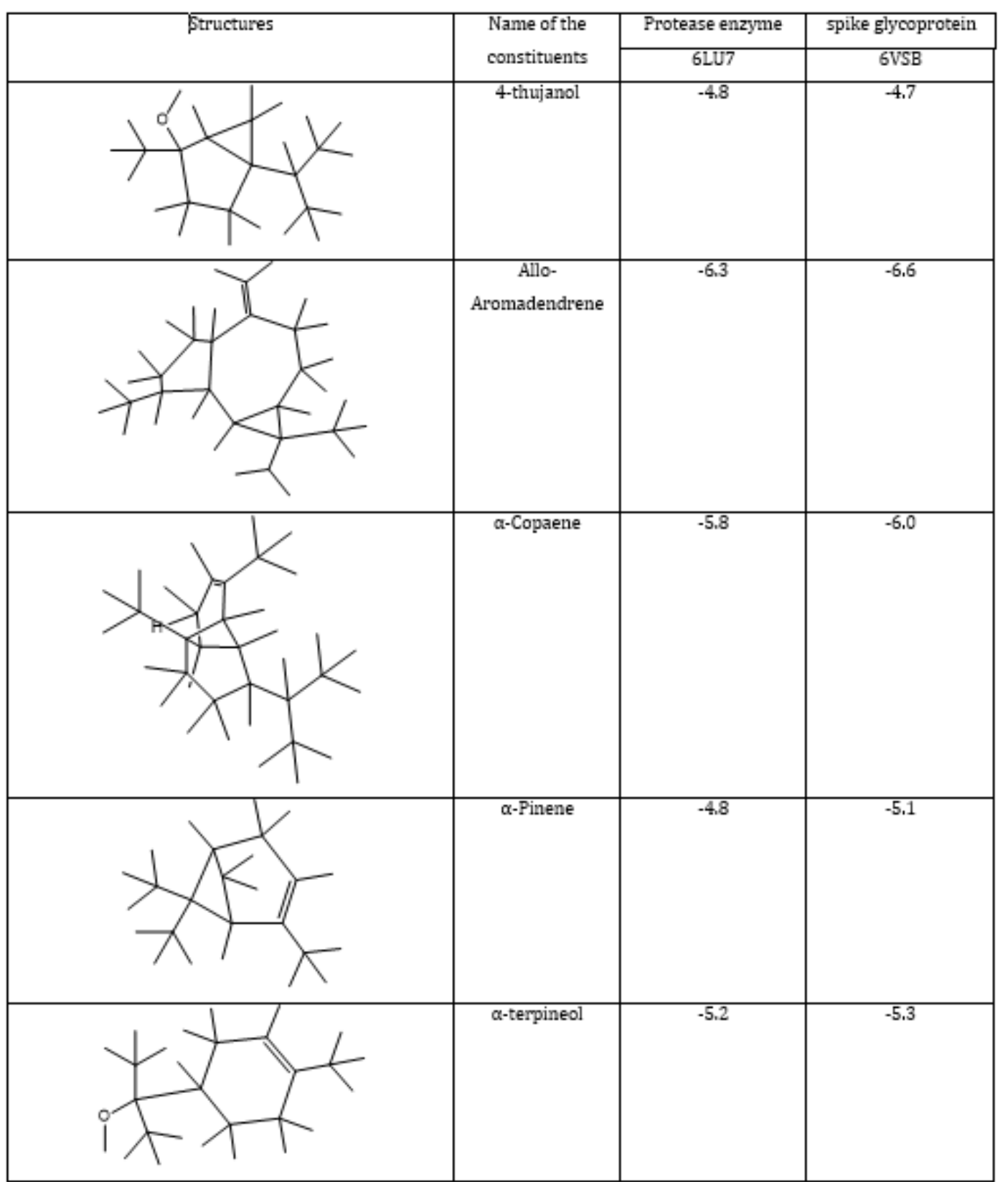



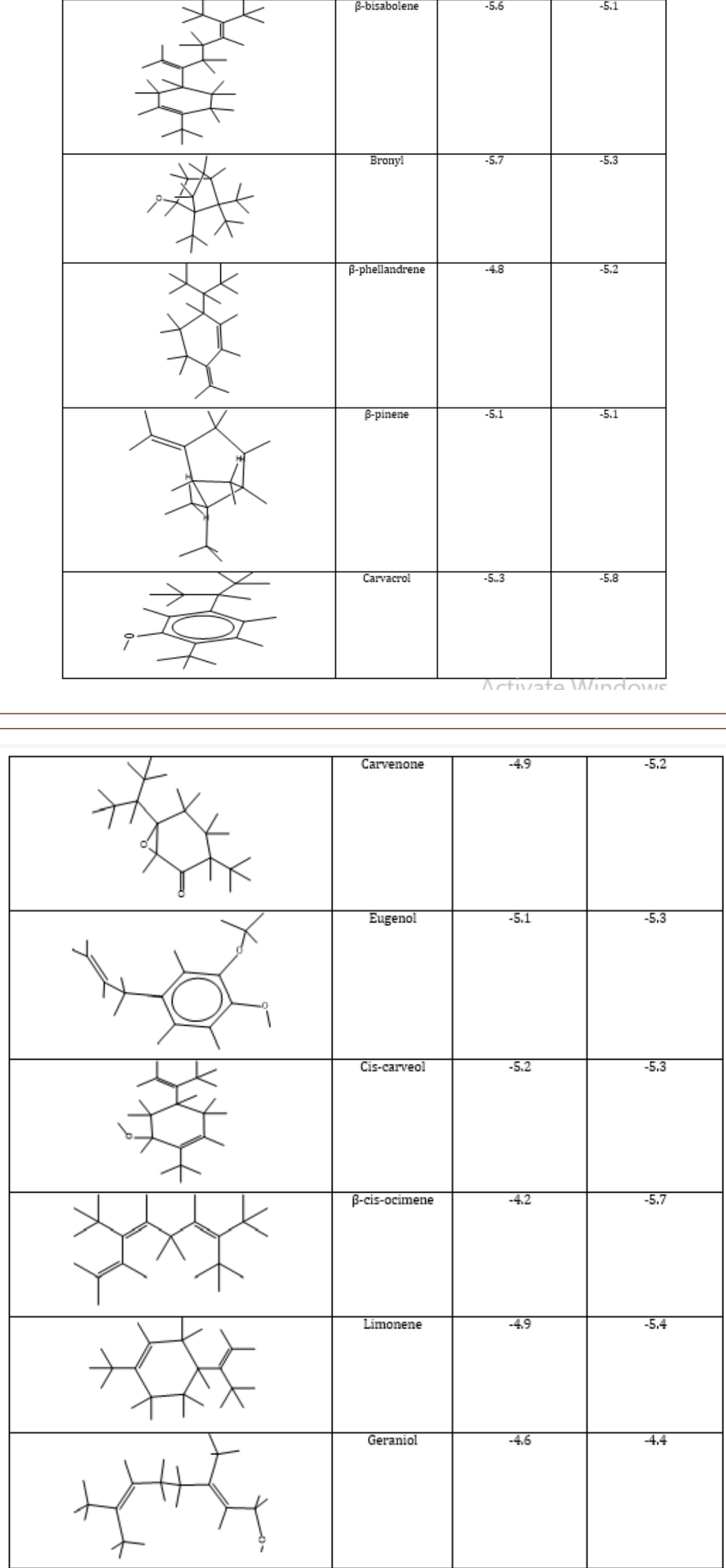

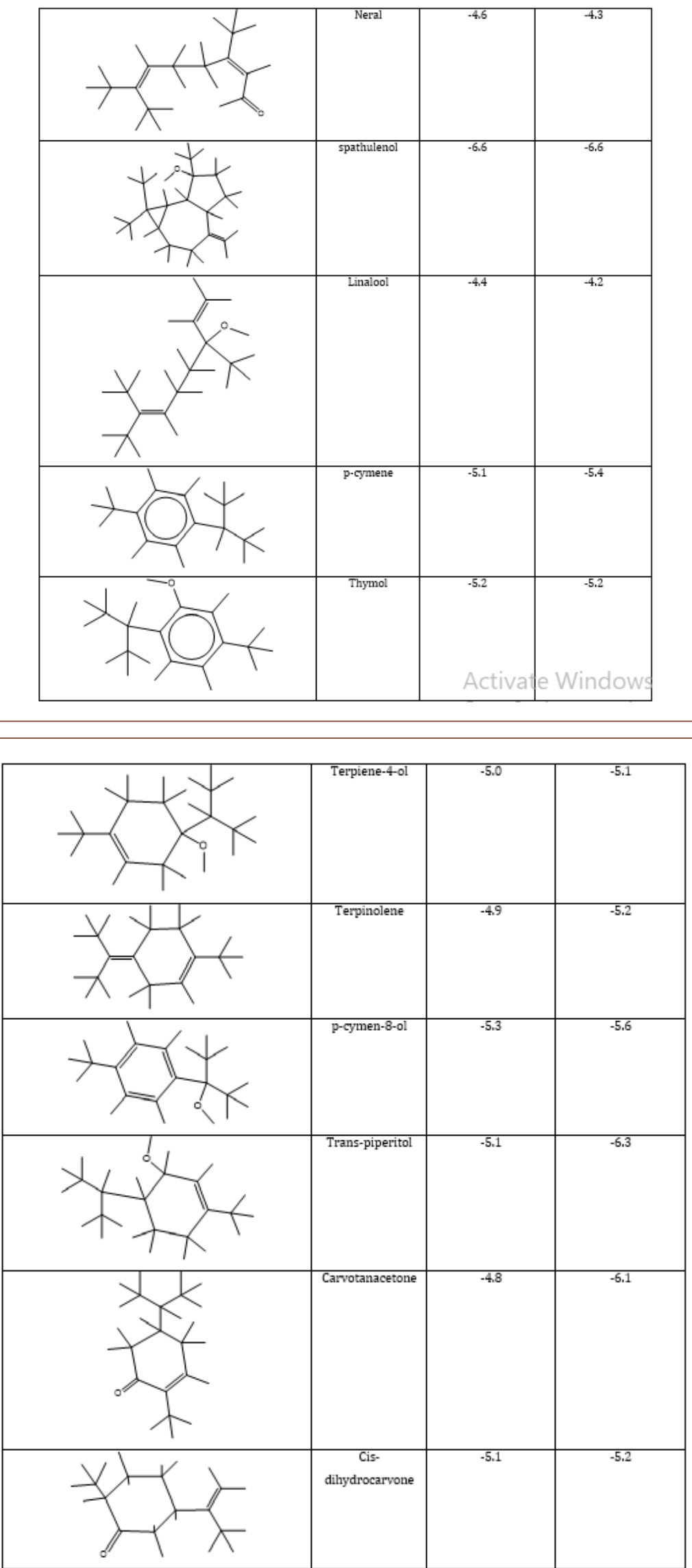


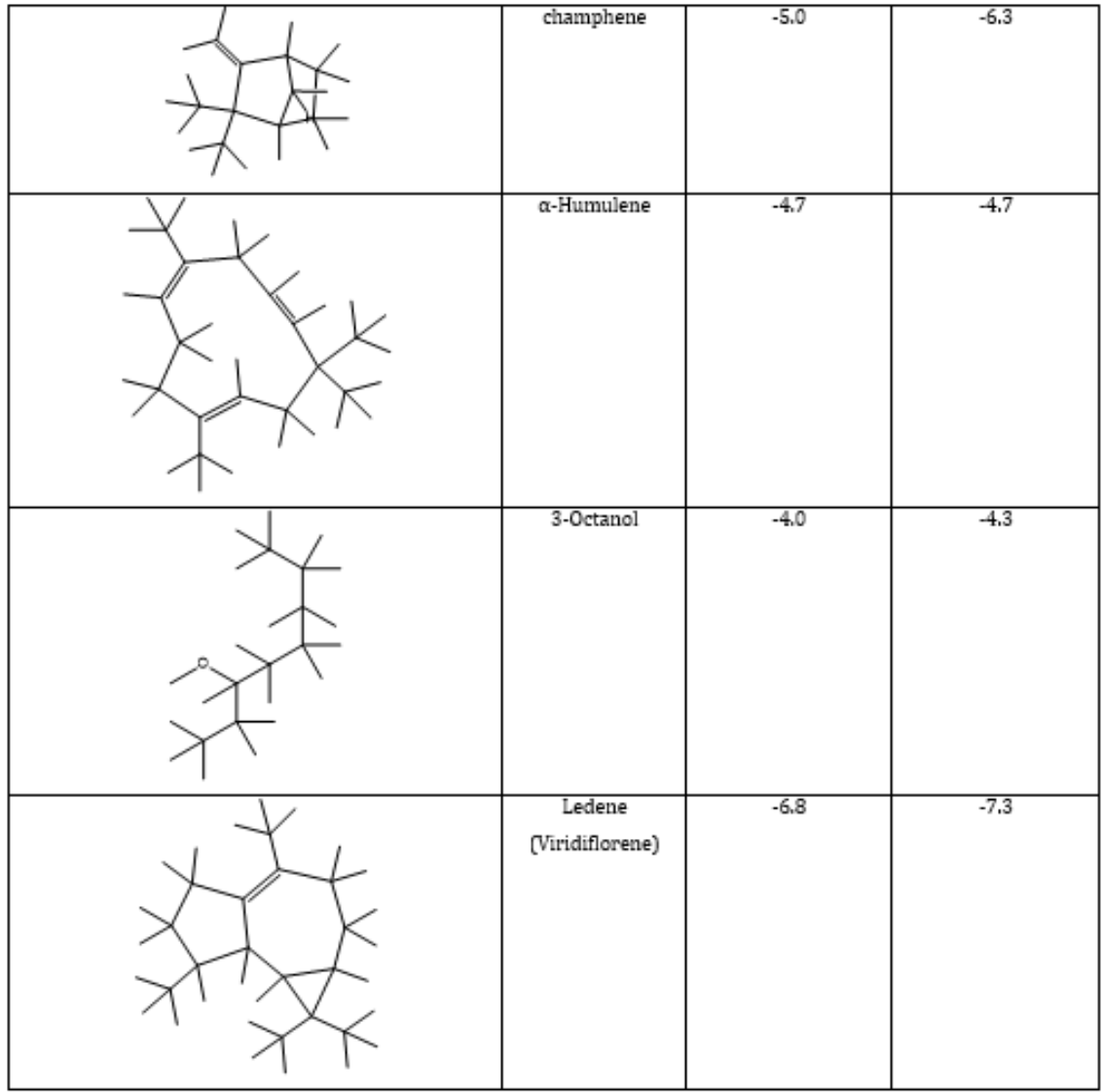

Table 1: Binding energies of Thymus capitatus constituents with protease enzyme (6LU7) and COVID-19 spike glycoprotein (6VSB) obtained from molecular dockings. The unit of all energies $(\Delta \mathrm{G})$ is $\mathrm{kcal} / \mathrm{mol}$.

The obtained results using computational drug repurposing offer an efficient way to find novel applications for already known drugs [75]. Molecular docking and binding free energy calculations for sesquiterpenes can be used to forecast drug-target interactions and binding affinity. The appearance of resistance to existing antiviral drugs or vaccines is a major challenge in antiviral drug development. The drug repurposing technique allows finding novel antiviral agents within a short period in order to overcome the challenges in antiviral therapy. Computational drug repurposing has previously been used to recognize drug candidates for viral infectious diseases like ZIKA, Ebola, influenza and dengue infections. These methods were also utilized to recognize possible drugs against MERS-CoV and SARS-CoV [76,77] and following the COVID-19 outbreak, computational repurposing can be used for finding COVID-19 antiviral agents. The Thymus capitatus sesquiterpenes are C15-terpenoids built from three isoprene units and are found mainly in higher plants as well as in fungi and marine organisms. Naturally, they are available as hydrocarbons or in oxygenated forms including alcohols, lactones, aldehydes, ketones and acids. Sesquiterpenes also include essential oils and aromatic constituents with several pharmacological activities. Studies report that sesquiterpenes have anti-inflammatory, anticancer, antidiabetic, antinociceptive and antimicrobial activities [78,79].
They also effect the central nervous system that make them possible targets for the development of novel therapeutics and of usage of medical intentions [78,79].

The results obtained by molecular modeling and by clinical use of Thymus capitatus extract are very promising also for fighting covid-19 viruses. It has been reported that sesquiterpenes directly inactivate herpes virus and might impede with virion envelope structures or mask viral structures that are essential for adsorption or entry into host cells. A virucidal activity of Melaleuca armillaris essential oil has been reported recently $[80,81]$ and dissolution of the herpes simplex virus envelope by treatment with oregano essential oil has been described [82]. Thus, different mechanisms of antiviral activity of many essential oils and compounds of essential oils including sesquiterpenes seem to be available. It has been also reported that Santolina insularis essential oil causes inactivation of herpesviruses and prevention of cell-to-cell spread [83,84]. Sesquiterpenes, were found to inhibit the severe acute respiratory syndrome caused by coronavirus [85,86], cytomegalovirus [87], and rhinovirus [88]. Furthermore, it has been reported that the chemical composition Thymus capitatus essential oil has antioxidative and antimicrobial activities [68,89]. These herbal species were also found to be contained in thymol and its isomer carvacrol, followed by their biosynthetic precursors, terpinene and 
$p$-cymene as main compounds [68,89]. In addition, literature data indicate that the powerful bioactive potential of Thymus capitatus constituents is principally related to the high proportion of phenolic compounds such as thymol and carvacrol [90]. Anti-inflammatory, antibacterial, antifungal, cytotoxic, insecticidal and herbicidal properties of thymol and carvacrol were studied previously [90].

In addition, numerous vaccines were recently developed by aiming at the exploration of vaccines to prevent and treat 2019. nCoV. Computational-based technology has given the researchers the tools to explore and identify potentially effective drug and/ or vaccine candidates which can efficiently shorten the time and diminish cost.

\section{Conclusion}

Essential oils and their components are known to be active against a wide variety of microorganisms. Many chemical constituents present in Thymus capitatus might contribute to antiviral activity. COVID-19 increasing oxidative stress, inflammations, and disturbance of the renin-angiotensin system, lead to damage, particularly the respiratory and the cardiovascular system. Thymus capitatus and its bioactive components suppress the negative effects of the renin-angiotensin system in COVID-19 patients. Moreover, many components of Thymus capitatus decrease pro-inflammatory cytokines such as IL- 6 and TNF- $\alpha$. Hence, it can decrease the severity of acute lung injury and the acute respiratory distress syndrome. Thymus capitatus extract reduces oxidative stress due to its antioxidant properties that can reduce adverse cardiovascular effects during COVID-19 infection. Finally, we conclude that Thymus capitatus has antioxidant, anti-inflammatory, and immunomodulating properties and diminishes side effects in COVID-19 patients as adjuvant.

\section{Acknowledgement}

The authors gratefully acknowledge the support and valuable suggestions obtained from Sir Khaled AB Diab (Judicial Expertise and Research Centre, Tripoli, Libya Tripoli, Libya).

\section{Conflict of Interest}

Author declare no conflict of interest.

\section{References}

1. Poursadeqiyan M, Bazrafshan E, Arefi MF (2020) Review of environmental challenges and pandemic crisis of Covid-19. J Educ Health Promot 9:250.

2. Ouassou H, Kharchoufa L, Bouhrim M, Daoudi NE, Imtara H, et al. (2020) The Pathogenesis of Coronavirus Disease 2019 (COVID-19): Evaluation and Prevention. J Immunol Res 2020: 1357983.

3. Wu J, Yuan X, Wang B, Gu R, Li W, et al. (2020) Severe Acute Respiratory Syndrome Coronavirus 2: From Gene Structure to Pathogenic Mechanisms and Potential Therapy. Front Microbiol 11: 1576

4. Rana AK, Rahmatkar SN, Kumar A, Singh D (2020) Glycogen synthase kinase-3: A putative target to combat severe acute respiratory syndrome coronavirus 2 (SARS-CoV-2) pandemic. Cytokine Growth Factor Rev 25: S1359-S6101.
5. Nishiga M, Wang DW, Han Y, Lewis DB, Wu JC. COVID-19 and cardiovascular disease: from basic mechanisms to clinical perspectives. Nat Rev Cardiol 17(9): 543-558.

6. Driggin E, Madhavan MV, Bikdeli B, Chuich T, Laracy J, et al. (2020) Cardiovascular Considerations for Patients, Health Care Workers, and Health Systems During the COVID-19 Pandemic. J Am Coll Cardiol 75(18): 2352-2371.

7. Otto WR, Geoghegan S, Posch LC, Bell LM, Coffin SE, et al. (2020) The Epidemiology of Severe Acute Respiratory Syndrome Coronavirus 2 in a Pediatric Healthcare Network in the United States. J Pediatric Infect Dis Soc 9(5): 523-529.

8. Baek WK, Sohn SY, Mahgoub A, Hage R (2020) A Comprehensive Review of Severe Acute Respiratory Syndrome Coronavirus 2. Cureus 12(5): e7943.

9. Keyhanian K, Umeton RP, Mohit B, Davoudi V, Hajighasemi F, et al. (2020) SARS-CoV-2 and nervous system: From pathogenesis to clinical manifestation. J Neuroimmunol 350: 577436

10. Ouassou H, Kharchoufa L, Bouhrim M, Daoudi NE, Imtara H, et al. (2020) The Pathogenesis of Coronavirus Disease 2019 (COVID-19): Evaluation and Prevention. J Immunol Res 2020: 1357983.

11. Han X, Wei X, Alwalid O, Cao Y, Li Y, et al. (2020) Severe Acute Respiratory Syndrome Coronavirus 2 among Asymptomatic Workers Screened for Work Resumption, China. Emerg Infect Dis 26(9): 2265-2267.

12. Wang Y, Liu Y, Liu L, Wang X, Luo N, et al. (2020) Clinical Outcomes in 55 Patients with Severe Acute Respiratory Syndrome Coronavirus 2 Who Were Asymptomatic at Hospital Admission in Shenzhen, China. J Infect Dis 221(11): 1770-1774.

13. Zhang J, Xu D, Xie B, Zhang Y, Huang H, et al. (2020) Poor-sleep is associated with slow recovery from lymphopenia and an increased need for ICU care in hospitalized patients with COVID-19: A retrospective cohort study. Brain Behav Immun 88: 50-58.

14. Khan AA, Ata F, Munir W, Yousaf Z (2020) Fluid Replacement Versus Fluid Restriction in COVID-19 Associated Hyponatremia. Cureus 12(7): e9059.

15. Zhang X, Alekseev K, Jung K, Vlasova A, Hadya N, et al. (2008) Cytokine Responses in Porcine Respiratory Coronavirus-Infected Pigs Treated with Corticosteroids as a Model for Severe Acute Respiratory Syndrome. J Virol 82(9): 4420-4428.

16. (2017) Diseases of the Respiratory System. Veterinary Medicine 8451090.

17. Hui DS, Zumla A (2019) Severe Acute Respiratory Syndrome: Historical, Epidemiologic, and Clinical Features. Infect Dis Clin North Am 33(4): 869-889.

18. Lin P, Wang M, Wei Y, Kim T, Wei X (2020) Coronavirus in human diseases: Mechanisms and advances in clinical treatment. MedComm (Beijing) 1:10

19. Hozhabri H, Piceci SF, Sohrabi H, Mousavifar L, Roy R, et al. (2020) The Global Emergency of Novel Coronavirus (SARS-CoV-2): An Update of the Current Status and Forecasting. Int J Environ Res Public Health 17(16): 5648

20. Liu S, Chan TC, Chu YT, Wu JTS, Geng X, et al. (2016) Comparative Epidemiology of Human Infections with Middle East Respiratory Syndrome and Severe Acute Respiratory Syndrome Coronaviruses among Healthcare Personnel. PLoS One 11(3): e0149988.

21. Sultana J, Cutroneo PM, Crisafulli S, Puglisi G, Caramori G, et al. (2020) Azithromycin in COVID-19 Patients: Pharmacological Mechanism, Clinical Evidence and Prescribing Guidelines. Drug Saf 43(8): 691-698.

22. Rosenberg ES, Dufort EM, Udo T, Wilberschied LA, Kumar J, et al. (2020) Association of Treatment with Hydroxychloroquine or Azithromycin with In-Hospital Mortality in Patients With COVID-19 in New York State. JAMA 323(24): 2493-2502. 
23. Felsenstein S, Herbert JA, McNamara PS, Hedrich CM (2020) COVID-19: Immunology and treatment options. Clin Immunol 215: 108448.

24. Zhang J, Xie B, Hashimoto K (2020) Current status of potential therapeutic candidates for the COVID-19 crisis. Brain Behav Immun 87: 59-73.

25. Jovic TH, Ali SR, Ibrahim N, Jessop ZM, Tarassoli SP, et al. (2020) Could Vitamins Help in the Fight Against COVID-19? Nutrients 12(9): 2550.

26. Xu Y, Baylink DJ, Chen CS, Reeves ME, Xiao J, et al. (2020) The importance of vitamin d metabolism as a potential prophylactic, immunoregulatory and neuroprotective treatment for COVID-19. J Transl Med 18(1): 322.

27. Xu Y, Baylink DJ, Chen CS, Reeves ME, Xiao J, et al. (2020) The importance of vitamin d metabolism as a potential prophylactic, immunoregulatory and neuroprotective treatment for COVID-19. J Transl Med 18(1): 322.

28. Khanna K, Kohli SK, Kaur R, Bhardwaj A, Bhardwaj V, et al. (2020) Herbal immune-boosters: Substantial warriors of pandemic Covid-19 battle. Phytomedicine: 153361.

29. Khalifa SA, Yosri N, El Mallah MF, Ghonaim R, Guo Z, et al. (2020) Screening for natural and derived bio-active compounds in preclinical and clinical studies: One of the frontlines of fighting the coronaviruses pandemic. Phytomedicine: 153311.

30. Ang L, Song E, Lee HW, Lee MS (2020) Herbal Medicine for the Treatment of Coronavirus Disease 2019 (COVID-19): A Systematic Review and Meta-Analysis of Randomized Controlled Trials. J Clin Med 9(5): 1583.

31. Afonso AF, Pereira OR, Cardoso SM (2020) Health-Promoting Effects of Thymus Phenolic-Rich Extracts: Antioxidant, Anti-inflammatory and Antitumoral Properties. Antioxidants (Basel) 9(9): 814.

32. Nieto G (2020) A Review on Applications and Uses of Thymus in the Food Industry. Plants (Basel) 9(8): 961.

33. Valencia Junca MA, Valencia C, Florez LE, Delgado-Ospina J, Zapata PA et al. (2019) Chitosan Beads Incorporated with Essential Oil of Thymus capitatus: Stability Studies on Red Tilapia Fillets. Biomolecules 9(9): 458.

34. El Abed N, Kaabi B, Smaali MI, Chabbouh M, Habibi K, et al. (2014) Chemical Composition, Antioxidant and Antimicrobial Activities of Thymus capitata Essential Oil with Its Preservative Effect against Listeria monocytogenes Inoculated in Minced Beef Meat. Evid Based Complement Alternat Med 2014: 152487.

35. Jaouadi R, Silva AMS, Boussaid M, Yahia IBH, Cardoso SM, et al. (2019) Differentiation of Phenolic Composition Among Tunisian Thymus algeriensis Boiss. et Reut. (Lamiaceae) Populations: Correlation to Bioactive Activities. Antioxidants (Basel) 8(11): 515

36. Afonso AF, Pereira OR, Cardoso SM (2020) Health-Promoting Effects of Thymus Phenolic-Rich Extracts: Antioxidant, Anti-inflammatory and Antitumoral Properties. Antioxidants (Basel) 9(9): 814

37. Nieto G (2020) A Review on Applications and Uses of Thymus in the Food Industry. Plants (Basel) 9(8): 961.

38. Valencia Junca MA, Valencia C, Florez LE, Delgado-Ospina J, Zapata PA, et al. (2019) Chitosan Beads Incorporated with Essential Oil of Thymus capitatus: Stability Studies on Red Tilapia Fillets. Biomolecules 9(9): 458.

39. Chowdhury S, Happonen L, Khakzad H, Malmstrom L, Malmstrom J (2020) Structural proteomics, electron cryo-microscopy and structural modeling approaches in bacteria-human protein interactions. Med Microbiol Immunol: 1-11.

40. John R, Arango-Jaramillo S, Self S, Schwartz DH (2004) Modeling partially effective HIV vaccines in vitro. J Infect Dis 189(4): 616-623.

41. Afonso AF, Pereira OR, Cardoso SM (2020) Health-Promoting Effects of Thymus Phenolic-Rich Extracts: Antioxidant, Anti-inflammatory and Antitumoral Properties. Antioxidants (Basel) 9(9): 814.

42. Verlinde CL, Merritt EA, Van den AF, Kim H, Feil I, et al. (1994) Protein crystallography and infectious diseases. Protein Sci 3(10): 1670-1686.
43. Tiwari R, Mahasenan K, Pavlovicz R, Li C, Tjarks W (2009) Carborane clusters in computational drug design: a comparative docking evaluation using AutoDock, FlexX, Glide, and Surflex. J Chem Inf Model 49(6): 15811589.

44. Abdul M Gbaj, Nisreen $\mathrm{H}$ Meiqal, Inass A Sadawe, Salah M Bensaber, Abdulathim A, et al. (2020) Docking Study of New OrthoPhenylenediamine Derivatives as COVID-19 Protease Inhibitors. Lupine online Journal of medical sciences 5(2): 471-475.

45. Youssefi MR, Tabari MA, Esfandiari A, Kazemi S, Moghadamnia AA, et al. (2019) Efficacy of Two Monoterpenoids, Carvacrol and Thymol, and Their Combinations against Eggs and Larvae of the West Nile Vector Culex pipiens. Molecules 24(10): 1867.

46. Mahran YF, Badr AM, Aldosari A, Bin-Zaid R, Alotaibi HN (2019) Carvacrol and Thymol Modulate the Crosstalk between TNF- $\alpha$ and IGF1 Signaling in Radiotherapy-Induced Ovarian Failure. Oxid Med Cell Longev 2019: 3173745.

47. Games E, Guerreiro M, Santana FR, Pinheiro NM, de Oliveira EA, et al (2016) Structurally Related Monoterpenes p-Cymene, Carvacrol and Thymol Isolated from Essential Oil from Leaves of Lippia sidoides Cham. (Verbenaceae) Protect Mice against Elastase-Induced Emphysema. Molecules 21(10): 1390.

48. Zamorano CN, Grandvaux N (2020) ACE2: Evidence of role as entry receptor for SARS-CoV-2 and implications in comorbidities. Elife 9: e61390.

49. Mussbacher M, Salzmann M, Brostjan C, Hoesel B, Schoergenhofer C, et al. (2019) Cell Type-Specific Roles of NF- $\kappa B$ Linking Inflammation and Thrombosis. Front Immunol 10: 85

50. Chen Z, Dong WH, Wu Q, Wang J (2020) Two-layer regulation of TRAF6 mediated by both TLR4/NF-kB signaling and miR-589-5p increases proinflammatory cytokines in the pathology of severe acute pancreatitis. Am J Transl Res 12(6): 2379-2395.

51. Mohan ML, Vasudevan NT, Naga Prasad SV (2017) Pro-inflammatory cytokines mediate GPCR dysfunction. J Cardiovasc Pharmacol 70(2): 6173.

52. Kuhla B (2020) Review: Pro-inflammatory cytokines and hypothalamic inflammation: implications for insufficient feed intake of transition dairy cows. Animal 14(S1): s65-s77.

53. Bukovská A, Cikos S, Juhás Å, Il’ková G, Rehák P, et al. (2007) Effects of a Combination of Thyme and Oregano Essential Oils on TNBS-Induced Colitis in Mice. Mediators Inflamm 2007: 23296.

54. Ustuner O, Anlas C, Bakirel T, Ustun-Alkan F, Diren SB, et al. (2019) In Vitro Evaluation of Antioxidant, Anti-Inflammatory, Antimicrobial and Wound Healing Potential of Thymus Sipyleus Boiss. Subsp. Rosulans (Borbas) Jalas. Molecules 24(18): 3353.

55. Khosravi AR, Erle DJ (2016) Chitin-Induced Airway Epithelial Cell Innate Immune Responses Are Inhibited by Carvacrol/Thymol. PLoS One 11(7): e0159459.

56. Ortiz-Prado E, Simbana-Rivera K, Barreno L, Rubio-Neira M, Guaman LP, et al. (2020) Clinical, molecular, and epidemiological characterization of the SARS-CoV-2 virus and the Coronavirus Disease 2019 (COVID-19), a comprehensive literature review. Diagn Microbiol Infect Dis 98(1): 115094

57. Vassiliou AG, Kotanidou A, Dimopoulou I, Orfanos SE (2020) Endothelial Damage in Acute Respiratory Distress Syndrome. Int J Mol Sci 21(22): 8793.

58. Matthay MA, Arabi YM, Siegel ER, Ware LB, Bos LDJ, et al. (2020) Phenotypes and personalized medicine in the acute respiratory distress syndrome. Intensive Care Med 46(12):2136-2152.

59. Khan MZ, Khan A, Xiao J, Ma J, Ma Y, et al. (2020) Overview of Research Development on the Role of NF-kB Signaling in Mastitis. Animals (Basel) 10(9): 1625.

60. Cui Z, Xie Z, Wang B, Zhong Z, Chen X, et al. (2015) Carvacrol protects neuroblastoma SH-SY5Y cells against Fe2+-induced apoptosis by 
suppressing activation of MAPK/JNK-NF-ÎoB signaling pathway. Acta Pharmacol Sin 36(12): 1426-1436.

61. Spalletta S, Flati V, Toniato E, Di Gregorio J, Marino A, et al. (2018) Carvacrol reduces adipogenic differentiation by modulating autophagy and ChREBP expression. PLoS One 13(11): e0206894.

62. Armas-Ricard M, Ruiz-Reyes E, Ramirez-Rodriguez O (2019) Caffeates and Caffeamides: Synthetic Methodologies and Their Antioxidant Properties. Int J Med Chem 2019: 2592609.

63. Boskabady MH, Alitaneh S, Alavinezhad A (2014) Carum copticum L.: A Herbal Medicine with Various Pharmacological Effects. Biomed Res Int 2014: 569087

64. Dhama K, Khan S, Tiwari R, Sircar S, Bhat S, et al. (2020) Coronavirus Disease 2019-COVID-19. Clin Microbiol Rev 33(4).

65. Nouri-Vaskeh M, Sharifi A, Khalili N, Zand R, Sharifi A (2020) Dyspneic and non-dyspneic (silent) hypoxemia in COVID-19: Possible neurological mechanism. Clin Neurol Neurosurg 198: 106217.

66. Coccimiglio J, Alipour M, Jiang ZH, Gottardo C, Suntres Z (2016) Antioxidant, Antibacterial, and Cytotoxic Activities of the Ethanolic Origanum vulgare Extract and Its Major Constituents. Oxid Med Cell Longev 2016: 1404505.

67. Afonso AF, Pereira OR, Neto RT, Silva AMS, Cardoso SM (2017) HealthPromoting Effects of Thymus herba-barona, Thymus pseudolanuginosus, and Thymus caespititius Decoctions. Int J Mol Sci 18(9): 1879.

68. Nishiga M, Wang DW, Han Y, Lewis DB, Wu JC. COVID-19 and cardiovascular disease: from basic mechanisms to clinical perspectives. Nat Rev Cardiol 17(9): 543-558.

69. Holt PA, Chaires JB, Trent JO (2008) Molecular docking of intercalators and groove-binders to nucleic acids using Autodock and Surflex. J Chem Inf Model 48(8): 1602-1615.

70. Afonso AF, Pereira OR, Cardoso SM (2020) Health-Promoting Effects of Thymus Phenolic-Rich Extracts: Antioxidant, Anti-inflammatory and Antitumoral Properties. Antioxidants (Basel) 9(9): 814

71. Gilad Y, Senderowitz H (2014) Docking studies on DNA intercalators. Chem Inf Model 54(1): 96-107.

72. Christianson DW (2017) Structural and Chemical Biology of Terpenoid Cyclases. Chem Rev 117(17): 11570-11648.

73. Gordaliza M (2012) Synthetic Strategies to Terpene Quinones/ Hydroquinones. Mar Drugs 10(2): 358-402.

74. Chen M, Al lami N, Janvier M, D’Antonio EL, Faraldos JA, et al. (2013) Mechanistic Insights from the Binding of Substrate and Carbocation Intermediate Analogues to Aristolochene Synthase. Biochemistry 52(32): 5441-5453.

75. Vanhaelen Q, Mamoshina P, Aliper AM, Artemov A, Lezhnina K, et al (2017) Design of efficient computational workflows for in silico drug repurposing. Drug Discov Today 22(2): 210-222.

76. Dyall J, Coleman CM, Hart BJ, Venkataraman T, Holbrook MR, et al. (2014) Repurposing of clinically developed drugs for treatment of Middle East respiratory syndrome coronavirus infection. Antimicrob Agents Chemother 58(8): 4885-4893.
77. Dyall J, Gross R, Kindrachuk J, Johnson RF, Olinger GG, et al. (2017) Middle East Respiratory Syndrome and Severe Acute Respiratory Syndrome: Current Therapeutic Options and Potential Targets for Novel Therapies. Drugs 77(18): 1935-1966.

78. Ambroz M, Bousova I, Skarka A, Hanusova V, Kralova V, et al. (2015) The Influence of Sesquiterpenes from Myrica rubra on the Antiproliferative and Pro-Oxidative Effects of Doxorubicin and Its Accumulation in Cancer Cells. Molecules 20(8): 15343-15358.

79. Di Sotto A, Mancinelli R, Gulli M, Eufemi M, Mammola CL, et al. (2020) Chemopreventive Potential of Caryophyllane Sesquiterpenes: An Overview of Preliminary Evidence. Cancers (Basel) 12(10): 3034.

80. Serafino A, Vallebona PS, Andreola F, Zonfrillo M, Mercuri L, et al. (2008) Stimulatory effect of Eucalyptus essential oil on innate cell-mediated immune response. BMC Immunol 9:17.

81. Astani A, Schnitzler P (2014) Antiviral activity of monoterpenes betapinene and limonene against herpes simplex virus in vitro. Iran J Microbiol 6(3): 149-155.

82. Astani A, Reichling J, Schnitzler P (2010) Comparative study on the antiviral activity of selected monoterpenes derived from essential oils. Phytother Res 24(5): 673-679.

83. da Silva JKR, Figueiredo PLB, Byler KG, Setzer WN (2020) Essential Oils as Antiviral Agents, Potential of Essential Oils to Treat SARS-CoV-2 Infection: An In-Silico Investigation. Int J Mol Sci 21(10): 3426.

84. Astani A, Reichling J, Schnitzler P (2011) Screening for Antiviral Activities of Isolated Compounds from Essential Oils. Evid Based Complement Alternat Med 2011: 253643.

85. Choudhry N, Zhao X, Xu D, Zanin M, Chen W, et al. (2020) Chinese Therapeutic Strategy for Fighting COVID-19 and Potential SmallMolecule Inhibitors against Severe Acute Respiratory Syndrome Coronavirus 2 (SARS-CoV-2). J Med Chem 63(22): 13205-13227.

86. Bhuiyan FR, Howlader S, Raihan T, Hasan M (2020) Plants Metabolites: Possibility of Natural Therapeutics Against the COVID-19 Pandemic. Front Med (Lausanne) 7: 444

87. Ramsey JT, Shropshire BC, Nagy TR, Chambers KD, Li Y, et al. (2020) Essential Oils and Health. Yale J Biol Med 93(2): 291-305.

88. Chen Y, Hamati E, Lee PK, Lee WM, Wachi S, Et al. (2006) Rhinovirus Induces Airway Epithelial Gene Expression through Double-Stranded RNA and IFN-Dependent Pathways. Am J Respir Cell Mol Biol 34(2): 192-203.

89. Liu Q, Meng X, Li Y, Zhao CN, Tang GY, et al. (2017) Antibacterial and Antifungal Activities of Spices. Int J Mol Sci 18(6): 1283.

90. Games E, Guerreiro M, Santana FR, Pinheiro NM, de Oliveira EA, et al (2016) Structurally Related Monoterpenes p-Cymene, Carvacrol and Thymol Isolated from Essential Oil from Leaves of Lippia sidoides Cham. (Verbenaceae) Protect Mice against Elastase-Induced Emphysema. Molecules 21(10): 1390. 